\title{
Towards the Development of a Navigation System for a Bipedal Robot: Preliminary Analysis of the Literature
}

\author{
Miguel Angel Ortega-Palacios, Josefina Guerrero-García, \\ Juan Manuel González-Calleros \\ Benemérita Universidad Autónoma de Puebla, \\ Language \& Knowledge Engineering (LKE), Puebla, Mexico \\ miguel.ortegapealumno.buap.mx, \\ \{josefina.guerrero, juan.gonzale\} @correo.buap.mx
}

\begin{abstract}
In the literature, several works are reported about the gait of bipedal robots. Some papers are associated with stability or automatic learning and navigation, but no paper was found that includes the variables of bipedal gait, stability, navigation and automatic learning, all applied in an indoor bipedal robot. Our objective is, after the analysis of the state of the art, to propose a methodology that will allow the autonomous navigation of a bipedal robot and validate its degree of autonomy with respect to the evaluation metrics reported in the literature.
\end{abstract}

Keywords: Bipedal Robot, Bipedal Gait, Stability, Navigation.

\section{Introduction}

As many mobile robots begin to integrate into different areas of society, they will need to operate in a wide variety of environments. Often, these environments will be dynamic; the objects move and the structures change physically. Less dynamic environments can be characterized by physical changes that occur over days, weeks or months, while more dynamic environments involve objects in continuous motion, such as human beings or vehicles.

Mobile robots cannot assume that the world is static if we expect them to work effectively. For a mobile robot to work autonomously in a dynamic environment, it must have a way of detecting its environment. Cameras are ubiquitous among modern robots and can provide a large amount of information about the environment. Robotics applications can employ techniques such as computer vision to perform object recognition, 3D reconstruction, mapping and localization tasks [1]. Nowadays, most robots require moving and performing tasks in a variety of environments that are sometimes even unpredictable. Navigation with mobile robots is a challenging problem in the field of robotics where numerous studies have been conducted that have resulted in a variety of solutions. Four integral parts are identified in the navigation of a biped robot: perception, location, movement control and trajectory planning.

The rest of this document is structured as follows: the second section addresses the importance of bipedal robots, section three presents the state of the art analysis, the 
limitations found in the literature are mentioned in the fourth section, the proposed methodology is presented in the fifth section and finally the conclusions are presented.

\section{Importance of Bipedal Robots}

The study of walking anthropomorphic robots has been a challenge for the scientific community until today, the fact to replicate the movements and the way of walking of human beings is not trivial and is something that has not been completely imitated.

Moreover, there is the inherent difficulty of giving artificial intelligence to a humanoid robot so that it is capable of learning to walk on its own in a dynamic environment, just as a human being does. Hence, the interest in developing an autonomous navigation system for a biped robot that is capable of navigating indoors through a horizontal plane, which can detect and evade static or mobile obstacles during its trajectory.

The advantages of this type of robots are not only limited to their ability to move on irregular terrain, but also have the advantage of having omnidirectional movement regardless of the orientation of its body. In addition, they have multiple applications that allow executing different tasks, such as surveillance, search, cooperation, transportation, navigation, and even carry out work that includes risks in the health or in the lives of people. In addition, the use of this type of robots can reduce execution times of some tasks, reducing fatigue in people, and therefore greater productivity when doing some task.

\section{State of the Art}

\subsection{Results of the Analysis of the Literature}

Among the works related to the navigation of mobile robots, most of the works are focused on robots with wheels [3-6, 9, 11-27], in second place the bipedal robots [8, 28-38] and in the last place are another type of robots which navigate on water or air $[2,7,10,39,40,41]$, as it can see in the graph of the Figure 1.

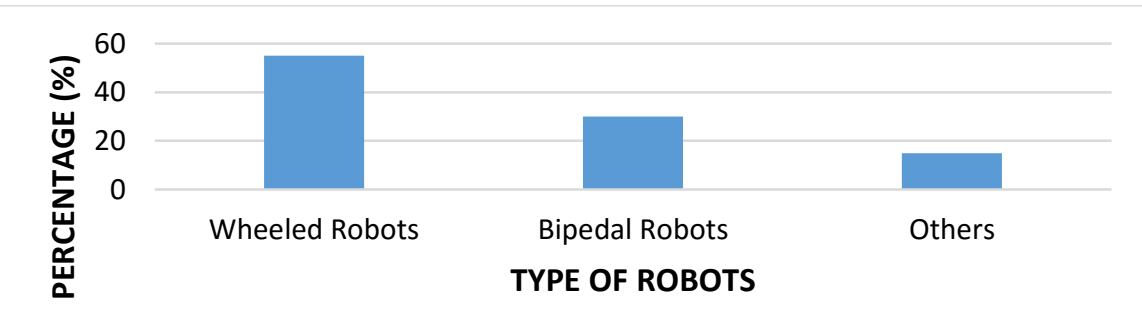

Fig. 1. Types of mobile robots studied in the literature. 
As can be identified in Figure 2, most of the works reviewed so far take into account navigation elements or navigation elements in conjunction with elements of machine learning, or only the variables of running and stability separately, but not found any that integrates all these concepts at the same time to develop an autonomous navigation system for bipedal robots.

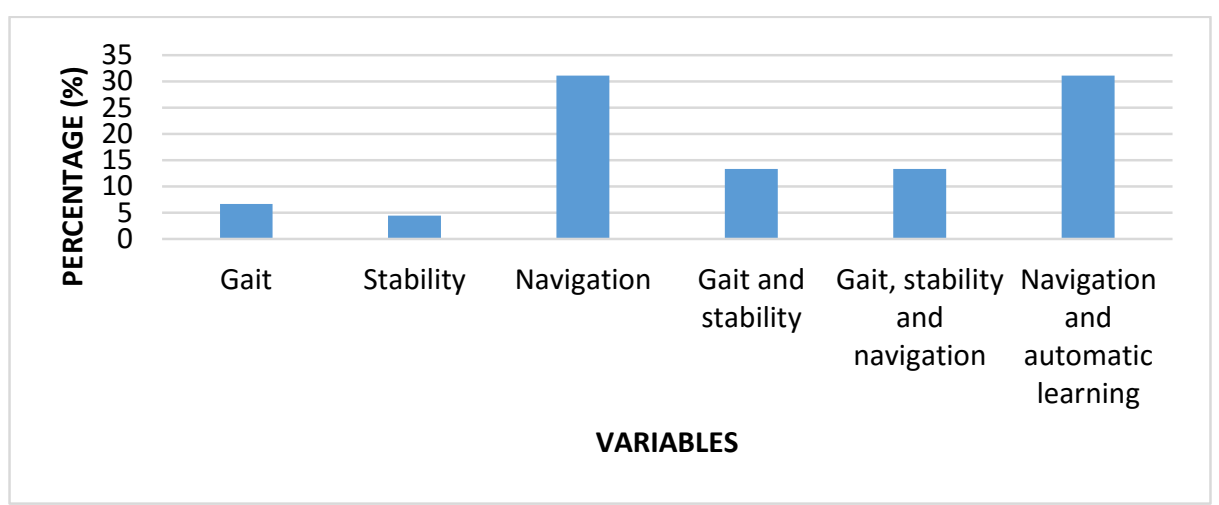

Fig. 2. Distribution of the study of the variables in the literature.

Table 1 identifies the works of the literature focused on the study of each of the variables: gait, stability, navigation and automatic learning.

As it can be observed, there are more researches reported that study stability in conjunction with navigation, so there are works that study navigation with the automatic learning, but it was not possible to find one that takes into account all the variables of interest.

Table 1. Variables in reviewed literature papers.

\begin{tabular}{|c|l|l|l|l|}
\hline Paper & Gait & Stability & Navigation & $\begin{array}{c}\text { Automatic } \\
\text { learning }\end{array}$ \\
\hline$[42],[43],[50]$ & & & & \\
\hline$[65],[78]$ & & & & \\
\hline $\begin{array}{c}{[44],[51],[52],[53],[54],} \\
{[55],[62],[64],[66],[69],} \\
{[70],[71],[68],[75]}\end{array}$ & & & & \\
\hline $\begin{array}{c}{[47],[49],[59],[60],[61],} \\
{[77]}\end{array}$ & & & & \\
\hline $\begin{array}{c}{[45],[56],[57],[58],[63],} \\
{[67]}\end{array}$ & & & & \\
\hline $\begin{array}{c}{[72],[73],[79],[80],[81],} \\
{[82],[83],[84],[85],[86],} \\
{[87],[88][89],[90]}\end{array}$ & & & & \\
\hline
\end{tabular}


There is a great variety of robots that are used to obtain the experimental results in the reviewed works of the literature, the most used reported in the investigations are the robots: Nao, Lola and HRP-2, as shown in Table 2.

Table 2. Robots used in literature papers.

\begin{tabular}{ccc}
\hline Robot & Paper & Percentage (\%) \\
\hline HRP-2 & {$[45],[63],[66],[80]$} & $9.52 \%$ \\
\hline Amber & {$[42]$} & $2.38 \%$ \\
\hline Nao & {$[42],[44],[52],[62],[64]$,} & $21.44 \%$ \\
\hline iCub & {$[69],[70],[75],[81]$} & $2.38 \%$ \\
\hline Lola & {$[53],[56],[57],[58],[59]$,} & $21.44 \%$ \\
\hline Lego mindstorm & {$[60],[61],[65],[67]$} & $2.38 \%$ \\
\hline Huro Evolution & {$[64]$} & $2.38 \%$ \\
\hline Tibi y Dabo & {$[71]$} & $2.38 \%$ \\
\hline SCITOS G5 & {$[73]$} & $2.38 \%$ \\
\hline Bioloid & {$[74],[77],[78]$} & $7.14 \%$ \\
\hline E-puck & {$[79]$} & $2.38 \%$ \\
\hline PR2 & {$[80]$} & $2.38 \%$ \\
\hline Kephera & {$[82],[83]$} & $4.76 \%$ \\
\hline Robot móvil & {$[85],[89]$} & $7.14 \%$ \\
\hline Robotino & {$[90]$} & $4.76 \%$ \\
\hline Robot Raving & {$[87]$} & $2.38 \%$ \\
\hline Daryl & & $2.38 \%$
\end{tabular}

As it can be observed, the Lola and Nao robot are the most used in the investigations, with respect to the Lola robot, its great use is due to the fact that there are multiple works belonging to the same authors, and they have followed it up to improve it through the years. While the use of the Nao robot is due to the fact that it is a robot with last generation technology, it has a high degree of interactivity for any type of user; it is fully programmable, and currently more than 400 universities are working on this platform. It makes this robot the platform with the most advanced technology in entertainment robotics for research and development.

Most of the works reviewed so far are focused on the detection and avoidance of obstacles in static or dynamic environments, that is, the obstacles can be fixed or can be moved during the navigation of the robot. In addition, the robots are regularly navigated on 1-level flat terrain [42, 44, 45, 47, 51, 54, 56-62, 64, 66, 68-73, 75, 77-90] or multi levels [52-67], even in irregular terrain [63]. The distribution of the type of land used in the experimentation of the reviewed papers is shown in Figure 3.

In the reviewed works, the application given to each robot is not specifically specified, only in [62] it is mentioned that the robot is intended to send a message to a staff member in an office environment, or for example in [68] the robot is required to be able to meet the technical challenge to avoid obstacles of the RoboCup Humanoid 
League. While in the other works there is the context of the need to navigate and avoid obstacles to reach a goal. Therefore, the context of the works is treated from a very general approach, as shown by the graph of Figure 4.

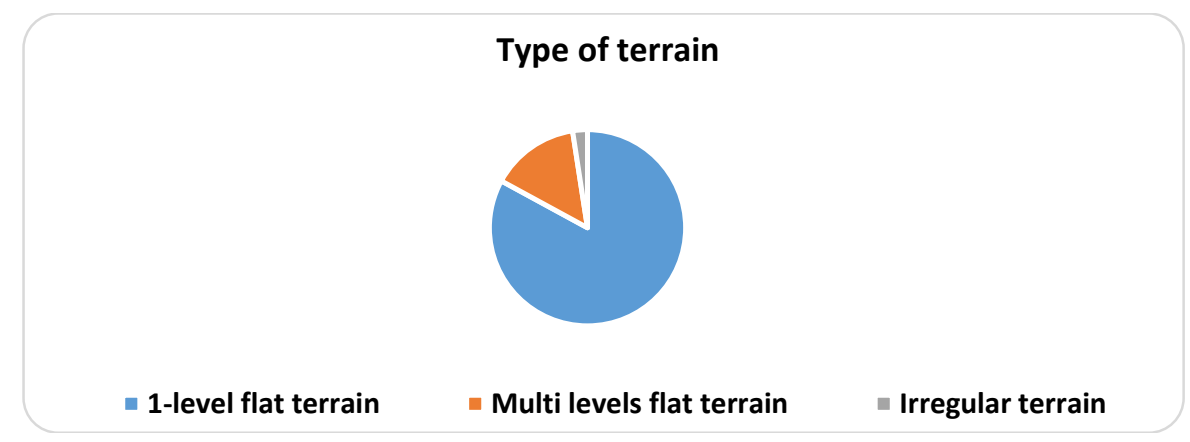

Fig. 3. Type of terrain used in the experimentation of the works reviewed in the literature

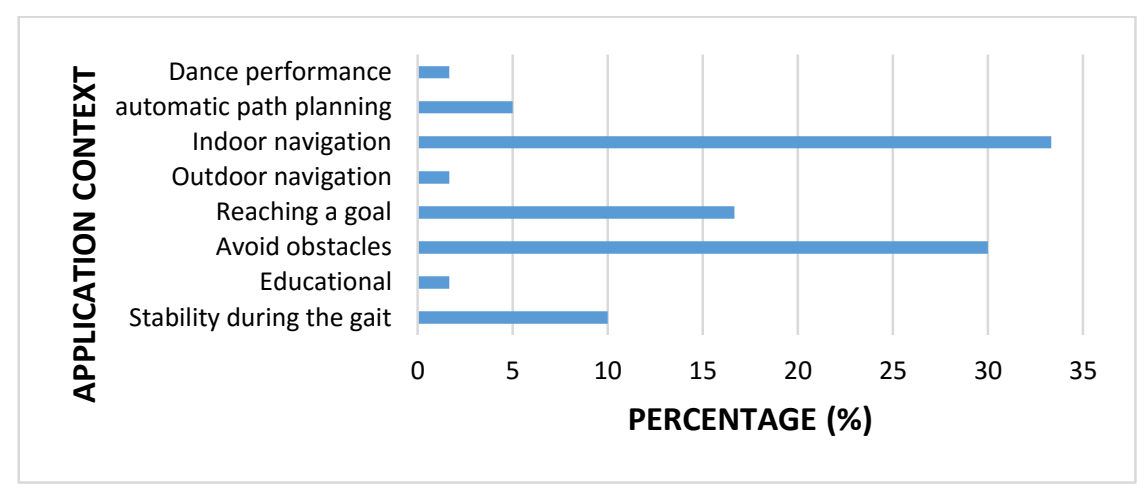

Fig. 4. Application context of the papers reviewed in the literature.

\section{$4 \quad$ Limitations Found in the Literature}

The main limitations found in the review of the literature are indicated below:

Physical restrictions of the robot. Due to the height of the robot, it is not always possible to capture good images of the reference points in the environment, because they could be placed at a height where the robot does not have access [62].

Restrictions on the movement of the robot. The planner only considers the quadrant directions (up, down, left, right) to prevent the robot from spinning while walking, which would increase the imprecision of the odometer data [62].

Computationally expensive. The computational resources required for pose estimation based on characteristics are quite expensive, and the method cannot be executed during the whole trip of the robot $[62,75]$. 
Use of a 3D map for the location. The location system may depend on the availability of a 3D map to locate, which must be acquired by the robot itself or provided by the user in advance [52, 57].

Fixed marks are used to locate the robot. The problem of localization is solved by using reference points placed by the user previously on the walls of the environment where the robot travels. It also remains a challenge to build more computationally efficient algorithms that can be used continuously to detect landmarks [62, 64].

Use of an external computer. The construction of the terrain map and the planning of the steps are carried out on a computer that is not mounted on the robot [63].

Wire data transmission. The sensor data and the step planning result are sent to / from the robot via Ethernet [63].

Use of the robot's predetermined functions. The functions that are already predefined in the programming of the robot are used, for example, a function for the recognition of objects or for the running of the robot [64].

The resolution of the images. The resolution of the images acquired by the camera is an important factor in defining the processing time of the camera, which can affect the efficiency of the algorithm if a real-time response is required [66, 68, 69, 81, 84].

Maximum speed of the robot. The time required for the robot to reach its goal will not only depend on the efficiency of the algorithm, but also on the maximum speed at which the robot can move $[58,67,70,74]$.

The size, shape, and texture of the obstacles. This is important, because the efficiency for the detection of obstacles depends very much on the size, texture or shape of the objects to be detected and also on the way in which the robot acquires the information of the environment, either through sensors or from a camera [52, 66].

Restricted field of view. The vision system, like most built-in vision systems, may fail to recognize reference points due to too large viewing angles (which significantly reduces the quality of the image) and variation in lighting conditions. In the case of sensors, depending on the type and its characteristics, it has a maximum and minimum distance for the detection of objects [52, 53, 58, 62, 79].

Limitation on the size of the test space. Experimental tests are carried out in usercontrolled environments, with either models or the use of harnesses so that the robot can move in a limited space $[57,83]$.

Static environment. The detection and avoidance of obstacles during the navigation of the robot only contemplates static obstacles and some are added in real time $[69,82$, $83]$.

\section{$5 \quad$ Proposed Methodology}

After performing this research in the literature; it is proposed to use a Bioloid Premium Robot with 18 degrees of freedom, since there is no access to a complete list of the samples that make up the population, a non-probabilistic sampling technique was used. In this case, convenience sampling was used, since a sample of the population was selected due to the fact that it was accessible, but not because it was selected by a statistical criterion. 
The study variables, presented graphically in Figure 5 are the following:

-Gait kinematics (independent), which corresponds to the analysis and modeling of the biped robot cycle.

-Stability (independent), which is related to the balance point of the robot to stand up during the march.

-Navigation (independent) will be defined so that the robot can move from one point to another in a horizontal plane.

-Level of autonomy (dependent), which will depend on the integration of the three previous variables so that the robot is able to navigate autonomously indoors, using an automatic learning algorithm.

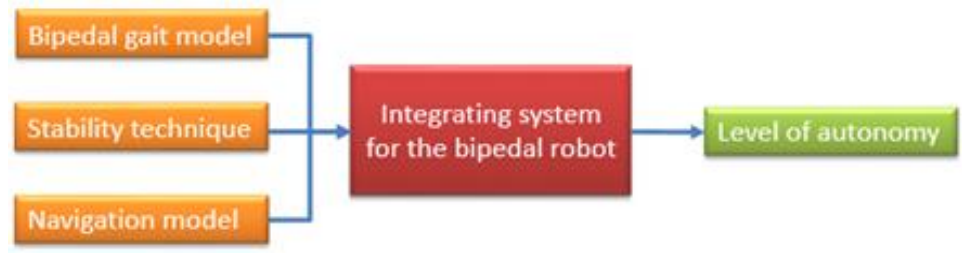

Fig. 5. System variables.

The proposed methodology aims to reduce the gap in the limitations related to wire data transmission, use of the robot's predefined functions and the detection of objects limited by their size, shape, and texture. In addition, this methodology will provide autonomy to the entire system, so that the control of each of the variables will be modified to obtain the best result at all times. The stages of the proposed methodology are:

-Study and analyze the operation of the robot. There will be an in-depth study of the Bioloid robot mechanism, analyzing its kinematics, that is, the limitations of the movements of each of its joints. Therefore, it is necessary to analyze the operation of the robot servomotors.

-Implement the human walking dynamics in the robot, using the dynamic model of the robot. As you can see in Figure 6.

Following the base of the scheme of Figure 7, the same process is applied to evaluate the methods of automatic learning, stability and navigation, continuing with the following steps of the methodology:

-Select and apply a stability method for the robot. The concept of zero moment point will be take into account; it is useful in determining if a bipedal robot is in a stable configuration or not. It has been used extensively in bipedal robots.

-Select and apply a navigation method for the robot. Different navigation methods will be evaluated, such as fuzzy control or reinforcement learning to develop automatic path planning, selecting the most satisfactory method.

-Implement all variables in the robot: gait, stability, navigation and automatic learning indoors.

-Validate the degree of autonomy in the navigation of the robot indoors. Evaluate the performance of the robot's automatic navigation, measuring the degree of autonomy of the robot, which is, making a comparison between the expected result and the real 
result, evaluating if the robot arrives satisfactorily at its goal in the expected time, acting as autonomous form. The criteria to determine the degree of autonomy can vary and will be attached to standardized metrics in the literature. If the result is not satisfactory, the entire process will have to be repeated.

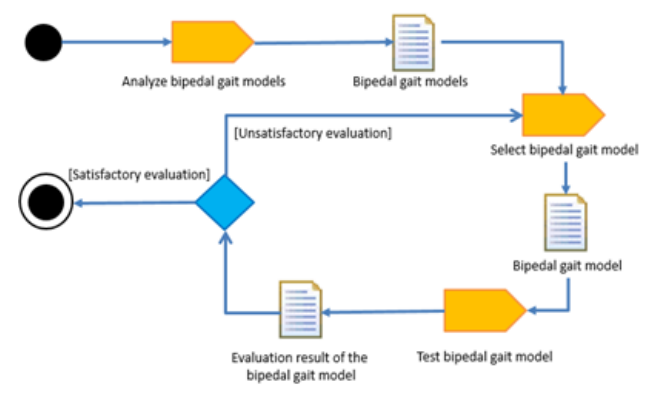

Fig. 6. Selection process of suitable gait model for the bipedal robot.

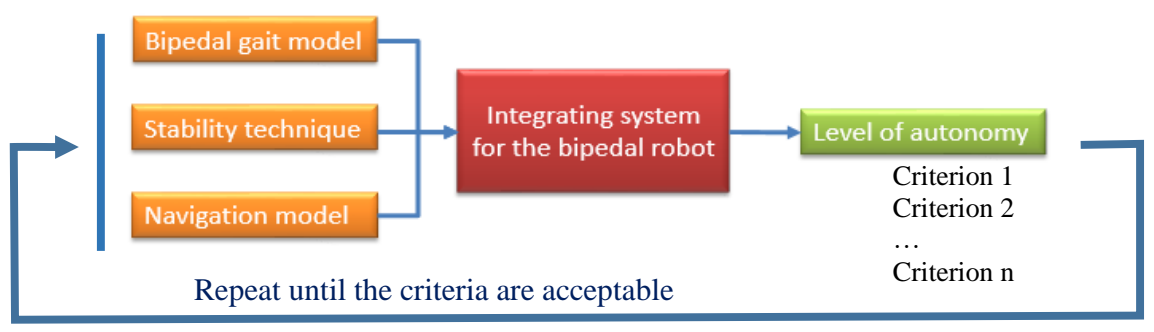

Fig. 7. Evaluation of the proposed solution based on criteria and parameters reported in the literature.

\section{Conclusions}

During the review of the literature, a work including all the study variables at the same time, i.e. bipedal gait, stability, navigation and automatic learning was not found. It is very important to design a methodology that integrates all the variables in a biped robot to be able to navigate autonomously indoors without using pre-established routines in order to generate a panorama of multiple applications for future researchers.

Regarding the study variables, most of the works are focused on the study of navigation and in conjunction with machine learning, but in this last approach only robots with wheels are used. It was also found that most of the robots are tested on flat terrain either one or more levels, probably because they do not include the study of the variable of stability in the case of bipedal robots, in the case of robots with wheels this does not apply since its function is to move on flat terrains. It is also simpler to avoid the use of stability in the autonomous navigation of bipedal robots when they have to walk on flat terrain. 
As mentioned above, the most used robots in the literature is the Nao and Lola robot. Another important point is that the specific application of each of the works reviewed in the literature is almost never mentioned, so it will be very important to find the specific context for which the proposed autonomous navigation system will be used and which will serve to justify your investigation.

Finally, it is important to point out that although there has been a lot of work in the area of navigation and bipedal robots. There are still many limitations that cause a high computational cost in the application of the algorithms, problems in acquiring environmental information in a correct manner, restrictions in the visual field of sensors, cameras or even restrictions on the physical characteristics of the robot itself.

\section{References}

1. Russell, S. J., Norvig, P.: Artificial Intelligence: a modern approach. Pearson Education Limited (2004)

2. Guan, X., Cai, C.: A New Integrated Navigation System for the Indoor Unmanned Aerial Vehicles (Uavs) Based on the Neural Network Predictive Compensation. In: 33th Youth Academic Annual Conference of Chinese Association of Automation (YAC), pp. 575-580 (2018)

3. Grewal, H. S., Jayaprakash, N. T., Matthews, A., Shrivastav, C., George, K.: Autonomous Wheelchair Navigation in Unmapped Indoor Environments. In: IEEE International Instrumentation and Measurement Technology Conference (I2MTC), pp. 1-6 (2018)

4. Catane, A., Feinberg, Y., Dor, O.: Indoor Navigation System. U. S. Patent Application Publication, Pub. No.: US 2013 / 0262223 A1 (2013)

5. Schtz, M., Dobrev, Y., Carlowitz, C., Vossiek, M.: Wireless Local Positioning with SILOBased Backscatter Transponders for Autonomous Robot Navigation. In: IEEE MTT-S International Conference on Microwaves for Intelligent Mobility (ICMIM), pp. 1-4 (2018)

6. Luo, R. C., Shih W.: Autonomous Mobile Robot Intrinsic Navigation Based on Visual Topological Map. In: IEEE 27th International Symposium on Industrial Electronics (ISIE), pp. 541-546 (2018)

7. Beak, D., Hwang, M., Kim, H., Kwon, D.: Path Planning for Automation of Surgery Robot Based on Probabilistic Roadmap and Reinforcement Learning. In: 15th International Conference, Ubiquitous Robot, pp. 342-347 (2018)

8. Mrudul, K., Mandava, R. K., Vundavilli, P. R.: An Efficient Path Planning Algorithm for Biped Robot Using Fast Marching Method. Procedia Comput. Sci. 133, 116-123 (2018)

9. Pattanayak, S., Sahoo, S. C., Choudhury, B. B.: An Effective Path Planning of a Mobile Robot. In: Soft Computing in Data Analytics, pp. 175-182 (2019)

10. Follestad M. H.: Autonomous Path-Planning and Following for a Marine Surface Robot. Master's thesis, Norwegian University of Science and Technology (2017)

11. Hsu, H. Y., Fu W., Lin Y.: Robot Surveillance System, U. S. Patent Application Publication, Pub. No.: US 2018 / 0181141 A1, (2018)

12. Fink, W., Brooks, A. J. W., Tarbell, M. A.: An Adaptive Hierarchical Approach to LidarBased Autonomous Robotic Navigation. Micro-Nanotechnology Sensors, Syst. Appl. X(69), 1-14 (2018)

13. Graven, O. H., Srisuphab, A., Silapachote, P., Sirilertworakul, V., Ampornwathanakun, W., Anekwiroj, P., Maitrichit, N.: An Autonomous Indoor Exploration Robot Rover and 3D Modeling with Photogrammetry. In: International ECTI Northern Section Conference on 
Electrical, Electronics, Computer and Telecommunications Engineering (ECTI-NCON), pp. 44-47 (2018)

14. Metka, B., Franzius, M., Bauer-Wersing, U.: Efficient Navigation Using Slow Feature Gradients. In: IEEE/RSJ International Conference on Intelligent Robots and Systems (IROS), pp. 1311-1316 (2017)

15. Kim, J. T., Choi, Y. H., Lee, J., Hong, S. H.: Floor-to-Floor Navigation for a Mobile Robot. In: 10th International Conference on Ubiquitous Robots and Ambient Intelligence (URAI), pp. 362-363 (2013)

16. Yun, C. H., Moon, Y. S., Ko, N. Y.: Vision Based Navigation for Golf Ball Collecting Mobile Robot. In: 13th International Conference on Control, Automation and Systems (ICCAS 2013), pp. 201-203 (2013)

17. Zhou, X., Weber, C., Wermter, S.: A Self-Organizing Method for Robot Navigation Based on Learned Place and Head-Direction Cells. In: 2018 International Joint Conference on Neural Networks (IJCNN), pp. 1-8 (2018)

18. Obo, T.: Intelligent Fuzzy Controller for Human-Aware Robot Navigation. In: 12th FranceJapan and 10th Europe-Asia Congress on Mechatronics, pp. 392-397. IEEE (2018)

19. Lim, J., Lee, S., Tewolde, G., Kwon, J.: Indoor Localization and Navigation for A Mobile Robot Equipped with Rotating Ultrasonic Sensors Using A Smartphone as The Robot's Brain. In: 2015 IEEE International Conference on Electro/Information Technology (EIT), pp. 621-625 (2015)

20. Chinnaaiah, M. C., Priyanka, G., Vani, G. D., Jyoti, M. A., Vennela, K.: A New Approach: An FPGA Based Robot Navigation for Patrolling in Service Environment. In: International Conference on Research Advances in Integrated Navigation Systems (RAINS), pp. 1-4. (2016)

21. Alves, P., Costelha, H., Neves, C.: Localization and Navigation of a Mobile Robot in an Office-Like Environment. In: 13th International Conference on Autonomous Robot Systems. pp. 1-6 (2013)

22. Le, C., Zhenghua, L.: Design of Two-Stage Fuzzy Controller for Mobile Robot Using Vision Navigation. In: Guidance, Navigation and Control Conference (CGNCC), 2016 IEEE Chinese. pp. 872-877 (2016)

23. Kim, T., Kim, J.: Beaconless Navigation for Mobile Robots Using Grid Line Pattern. In: Ubiquitous Robots and Ambient Intelligence (URAI), 2013 10th International Conference on, pp. 200-205 (2013)

24. Ko, B., Choi, H. J., Hong, C., Kim, J. H., Kwon, O. C., Yoo, C. D.: Neural Network-Based Autonomous Navigation for a Homecare Mobile Robot. In: Big Data and Smart Computing (Bigcomp), 2017 IEEE International Conference on Big Data, pp. 403-406 (2017)

25. Ravankar, A. A., Ravankar, A., Emaru, T., Kobayashi, Y.: A Hybrid Topological Mapping and Navigation Method for Large Area Robot Mapping. In: 56th Annual Conference of Society of Instrument and Control Engineers of Japan (SICE), pp. 1104-1107. (2017)

26. Novitsky, A., Yukhimets, D.: The Navigation Method of Wheeled Mobile Robot Based on Data Fusion Obtained from Onboard Sensors and Camera. In: 15th International Conference on Control, Automation and Systems (ICCAS), pp. 574-579 (2015)

27. Crépon, P. A., Panchea, A., Chapoutot, A.: Reliable Navigation Planning Implementation on a Two-Wheeled Mobile Robot. In: Second IEEE International Conference on Robotic Computing (IRC), pp. 173-174 (2018)

28. Mufti, S., Alam, M. N.: Real-Time Obstacle Avoidance and Path Planning of a SelfNavigated Autonomous Biped Robot Using Rugbat Sonar Sensors and Modular Digital Image Processing. In: 2012 International Conference on Robotics and Artificial Intelligence (ICRAI), pp. 75-80 (2012) 
29. Elmogy, M., Habel, C., Zhang, J.: Online Motion Planning for Hoap-2 Humanoid Robot Navigation. In: International Conference on Intelligent Robots and Systems, pp. 3531-3536 (2009)

30. Gutmann, J. S., Fukuchi, M., Fujita, M.: A Floor and Obstacle Height Map for 3D Navigation of a Humanoid Robot. In: Proceedings of the 2005 IEEE International Conference on Robotics and Automation, pp. 1066-1071 (2005)

31. Wahrmann, D., Hildebrandt, A. C., Wittmann, R., Sygulla, F., Rixen, D., Buschmann, T.: Fast Object Approximation for Real-Time 3D Obstacle Avoidance with Biped Robots. In: 2016 IEEE International Conference on Advanced Intelligent Mechatronics (AIM), pp. 3845 (2016)

32. Brooks, G., Krishnamurthy, P., Khorrami, F.: A Multi-Gait Approach for Humanoid Navigation in Cluttered Environments. In: The $26^{\text {th }}$ Chinese Control and Decision Conference (2014 CCDC), pp. 2708-2713 (2014)

33. Arias, L. E., Olvera, L. I., Pámanes, J. A., Núñez, J. V.: Patrón De Marcha 3D de Tipo Cicloidal para Humanoides y su Aplicación al Robot Bioloid. Revista Iberoamericana de Ingeniería Mecánica, 18(1), 3-22 (2014)

34. Fierro, J., Pámanes, J. A., Santibanez, V., Ruiz, G., Ollervides, J.: Condiciones para una Marcha Elemental del Robot NAO. Amrob J. Rob. Theor. Appl, 4(1), 13-18 (2014)

35. De Robótica, A. M., Industria, A. C.: Movimientos de un Robot Humanoide para el Pateado de un Balón de Fútbol. AMRob Journal, Robotics: Theory and Applications 3(2) 105-112 (2015)

36. González, J. J. T., Magé, H. F. N., Rodas, C. F. R.: Simulación del Ciclo de Marcha del Robot Bioloid en el Entorno Virtual V-Rep. In: Memorias del XVII Congreso Mexicano de Robótica 2015, pp. 1-8 (2015)

37. Brenes, J. C., Blanes, F.: Interfaz de Programación de Aplicaciones para Locomoción de Robot Humanoide Bioloid. Tésis de maestría, Unversidad Politécnica de Valencia (2016)

38. Cuperman, D., Verner, I. M.: Fostering Analogical Reasoning Through Creating Robotic Models of Biological Systems. Journal of Science Education and Technology 28(2), 90-103 (2018)

39. Qi, X., Chen, L., An, K., Wang, J., Zhang, B., Xu, K., Ye, D., Li C., Ran, L.: Bio-Inspired in-Grid Navigation and Positioning Based on an Artificially Established Magnetic Gradient. IEEE Transactions on Vehicular Technology 67(11), 10583-10589 (2018)

40. Somov, Y. I., Butyrin, S. A., Somov, S. Y.: Navigation, Guidance and Control of a FreeFlying Robot During its Rendezvous with a Passive Space Vehicle. In: 25th Saint Petersburg International Conference on Integrated Navigation Systems (ICINS), pp. 1-5. (2018)

41. Li, H., Savkin, A. V.: A Method for Collision Free Sensor Network Based Navigation of Flying Robots Among Moving and Steady Obstacles. In: 36th Chinese Control Conference (CCC), pp. 6079-6083 (2017)

42. Ames, A. D.: Human-Inspired Control of Bipedal Walking Robots. IEEE Transactions on Automatic Control 59(5), 1115-1130 (2014)

43. Vasudevan, R., Ames, A., Bajcsy, R.: Persistent Homology for Automatic Determination of Human-Data Based Cost of Bipedal Walking. Nonlinear Analysis: Hybrid Systems 7(1), 101-115 (2013)

44. Wen, S., Chen, X., Ma, C., Lam, H. K., Hua, S.: The Q-Learning Obstacle Avoidance Algorithm Based on EKF-SLAM for NAO Autonomous Walking under Unknown Environments. Robotics and Autonomous Systems 72, 29-36 (2015)

45. Nishiwaki, K., Chestnutt, J., Kagami, S.: Planning and Control of a Humanoid Robot for Navigation on Uneven Multi-Scale Terrain. In Experimental Robotics, pp. 401-415. Springer, Berlin, Heidelberg (2014) 
46. Nolfi, S., Bongard, J., Husbands, P., Floreano, D.: Evolutionary Robotics. In: Springer Handbook of Robotics, pp. 2035-2068. Springer (2016)

47. Ibanez, A., Bidaud, P., Padois, V.: Automatic Optimal Biped Walking as a Mixed-Integer Quadratic Program. In: Advances in Robot Kinematics, pp. 505-516. Springer (2014)

48. Todd, D. J.: Walking Machines: An Introduction to Legged Robots. Springer Science \& Business Media (2013)

49. Choi, Y. J., Kim, D. I., Oh, Y. H., Kim, C. H., You, B. J., San Cho, J.: U.S. Patent No. 8,738,178. Washington, DC: U.S. Patent And Trademark Office (2014)

50. Brasseur, C., Sherikov, A., Collette, C., Dimitrov, D., Wieber, P. B.: A Robust Linear MPC Approach to Online Generation of 3D Biped Walking Motion. In: 15th International Conference on Humanoid Robots (Humanoids), pp. 595-601 (2015)

51. Ha, E. T., Kim, T. K., Ahn, D. K., Jeong, S. H., Yoon, I. R., Han, S. H.: A Stable Control of Legged Robot Based on Ultrasonic Sensor. In: 15th International Conference on Control, Automation and Systems (ICCAS), pp. 1256-1258 (2015)

52. Hornung, A., Oßwald, S., Maier, D., Bennewitz, M.: Monte Carlo Localization for Humanoid Robot Navigation in Complex Indoor Environments. International Journal of Humanoid Robotics 11(2), 1-27 (2014)

53. Hildebrandt, A. C., Klischat, M., Wahrmann, D., Wittmann, R., Sygulla, F., Seiwald, P., Buschmann, T.: Real-Time Path Planning in Unknown Environments for Bipedal Robots. IEEE Robotics and Automation Letters 2(4), 1856-1863 (2017)

54. Mac Thi, T., Copot, C., Ionescu, C. M.: Design and Implementation of a Real-Time Autonomous Navigation System Applied to Lego Robots. In: 3rd IFAC Conference on Advances in Proportional-Integral-Derivative Control, pp. 340-345 (2018)

55. Tai, L., Zhang, J., Liu, M., Boedecker, J., Burgard, W.: A Survey of Deep Network Solutions for Learning Control in Robotics: From Reinforcement to Imitation. Arxiv Preprint Arxiv:1612.07139 (2019)

56. Hildebrandt, A. C., Demmeler, M., Wittmann, R., Wahrmann, D., Sygulla, F., Rixen, D., Buschmann, T.: Real-Time Predictive Kinematic Evaluation and Optimization for Biped Robots. In: International Conference on Intelligent Robots and Systems (IROS), pp. 57895796 (2016)

57. Hildebrandt, A. C., Wittmann, R., Wahrmann, D., Ewald, A., Buschmann, T.: Real-Time 3D Collision Avoidance for Biped Robots. In: International Conference on Intelligent Robots and Systems, pp. 4184-4190 (2014)

58. Wahrmann, D., Hildebrandt, A. C., Wittmann, R., Sygulla, F., Rixen, D., Buschmann, T.: Fast Object Approximation for Real-Time 3D Obstacle Avoidance with Biped Robots. In: IEEE International Conference on Advanced Intelligent Mechatronics (AIM), pp. 38-45 (2016)

59. Wittmann, R., Hildebrandt, A. C., Wahrmann, D., Sygulla, F., Rixen, D., Buschmann, T.: Model-Based Predictive Bipedal Walking Stabilization. In: Humanoids, pp. 718-724 (2016)

60. Wittmann, R., Hildebrandt, A. C., Ewald, A., Buschmann, T.: An Estimation Model for Footstep Modifications of Biped Robots. In: International Conference on Intelligent Robots and Systems (IROS 2014), 2014 IEEE/RSJ, pp. 2572-2578 (2014)

61. Wittmann, R., Hildebrandt, A. C., Wahrmann, D., Rixen, D., Buschmann, T.: State Estimation for Biped Robots Using Multibody Dynamics. In: International Conference on Intelligent Robots And Systems (IROS), 2015 IEEE/RSJ, pp. 2166-2172 (2015)

62. Wei, C., Xu, J., Wang, C., Wiggers, P., Hindriks, K.: An Approach to Navigation for the Humanoid Robot Nao in Domestic Environments. In: Conference Towards Autonomous Robotic Systems, pp. 298-310 (2013) 
63. Nishiwaki, K., Chestnutt, J., Kagami, S.: Autonomous Navigation of a Humanoid Robot over Unknown Rough Terrain. In: Robotics Research, pp. 619-634 (2017)

64. Wen, S., Othman, K. M., Rad, A. B., Zhang, Y., Zhao, Y.: Indoor SLAM Using Laser and Camera with Closed-Loop Controller for NAO Humanoid Robot. In: Abstract and Applied Analysis, pp 1-9 (2014)

65. Wahrmann, D., Knopp, T., Wittmann, R., Hildebrandt, A. C., Sygulla, F., Seiwald, P., Buschmann, T.: Modifying the Estimated Ground Height to Mitigate Error Effects on Bipedal Robot Walking. In: International Conference on Advanced Intelligent Mechatronics (AIM), pp. 1471-1476 (2017)

66. Kita, N., Kanehiro, F., Morisawa, M., Kaneko, K.: Obstacle Detection for a Bipedal Walking Robot by a Fisheye Stereo. In: International Symposium on System Integration (SII), pp. 119-125 (2013)

67. Wahrmann, D., Hildebrandt, A. C., Bates, T., Wittmann, R., Sygulla, F., Seiwald, P., Rixen, D.: Vision-Based 3D Modeling of Unknown Dynamic Environments for Real-Time Humanoid Navigation. International Journal of Humanoid Robotics 16(1), 1-34 (2019)

68. Kuo, C. H., Chou, H. C., Chi, S. W., Lien, Y. D.: Vision-Based Obstacle Avoidance Navigation with Autonomous Humanoid Robots for Structured Competition Problems. International Journal of Humanoid Robotics 10(3), 1-36 (2013)

69. Fakoor, M., Kosari, A., Jafarzadeh, M.: Revision on Fuzzy Artificial Potential Field for Humanoid Robot Path Planning in Unknown Environment. International Journal of Advanced Mechatronic Systems 6(4), 174-183 (2015)

70. Fakoor, M., Kosari, A., Jafarzadeh, M.: Humanoid Robot Path Planning with Fuzzy Markov Decision Processes. Journal of Applied Research and Technology 14(5), 300-310 (2016)

71. Goldhoorn, A., Garrell, A., Alquézar, R., Sanfeliu, A.: Continuous Real Time POMCP to Find-and-Follow People by a Humanoid Service Robot. In: International Conference on Humanoid Robots (Humanoids), pp. 741-747 (2014)

72. Motlagh, O., Nakhaeinia, D., Tang, S. H., Karasfi, B., Khaksar, W.: Automatic Navigation of Mobile Robots in Unknown Environments. Neural Computing and Applications 24(7), 1569-1581 (2014)

73. Dash, T.: Automatic Navigation of Wall Following Mobile Robot Using Adaptive Resonance Theory of Type-1. Biologically Inspired Cognitive Architectures 12, 1-8 (2015)

74. Han, J. K., Ha, I. Y., Kim, B. S.: Educational Robotic Construction Kit: Bioloid. In: 17th World Congress of The International Federation of Automatic Control (IFAC 2008), pp. 610 (2008)

75. George, L., Mazel, A.: Humanoid Robot Indoor Navigation Based on 2D Bar Codes: Application to the NAO Robot. In: 13th International Conference on Humanoid Robots (Humanoids), pp. 329-335 (2013)

76. Pandey, A., Pandey, S., Parhi, D. R.: Mobile Robot Navigation and Obstacle Avoidance Techniques: a Review. International Robotics \& Automation Journal 2(3), 1-12 (2017)

77. Meggiolaro, M. A., Neto, M. S., Figueroa, A. L.: Modeling and Optimization with Genetic Algorithms of Quasi-Static Gait Patterns in Planar Biped Robots. In: Congreso Internacional de Ingeniería Mecatrónica y Automatización (CIIMA 2016), pp. 1-10 (2016)

78. Segura, C. G., García, M. G., Díaz, L. N., Pech, R. B.: Robot Bioloid Premium Jaranero Controlado Remotamente por Voz. Pistas Educativas 39(126), 99-127 (2017)

79. Fathinezhad, F., Derhami, V., Rezaeian, M.: Supervised Fuzzy Reinforcement Learning for Robot Navigation. Applied Soft Computing 40, 33-41 (2016)

80. Furuta, Y., Wada, K., Murooka, M., Nozawa, S., Kakiuchi, Y., Okada, K., Inaba, M.: Transformable Semantic Map Based Navigation Using Autonomous Deep Learning Object 
Segmentation. In: 16th International Conference On Humanoid Robots (Humanoids), pp. 614-620. (2016)

81. Lobos-Tsunekawa, K., Leiva, F., Ruiz-Del-Solar, J.: Visual Navigation For Biped Humanoid Robots Using Deep Reinforcement Learning. IEEE Robotics and Automation Letters 3(4), 3247-3254 (2018)

82. Konar, A., Chakraborty, I. G., Singh, S. J., Jain, L. C., Nagar, A. K.: A Deterministic Improved Q-Learning for Path Planning of a Mobile Robot. IEEE Transactions on Systems, Man, and Cybernetics: Systems 43(5), 1141-1153 (2013)

83. Algabri, M., Mathkour, H., Ramdane, H., \& Alsulaiman, M.: Comparative Study of Soft Computing Techniques for Mobile Robot Navigation in an Unknown Environment. Computers in Human Behavior 50, 42-56 (2015)

84. Ran, L., Zhang, Y., Zhang, Q., Yang, T.: Convolutional Neural Network-Based Robot Navigation Using Uncalibrated Spherical Images. Sensors 17(6), 1-18 (2017)

85. Bischoff, B., Nguyen-Tuong, D., Lee, I. H., Streichert, F., Knoll, A.: Hierarchical Reinforcement Learning For Robot Navigation. In: Proceedings of The European Symposium on Artificial Neural Networks, Computational Intelligence And Machine Learning (ESANN 2013), pp. 227-232 (2013)

86. Bacciu, D., Gallicchio, C., Micheli, A., Di Rocco, M., Saffiotti, A.: Learning Context-Aware Mobile Robot Navigation in Home Environments. In: 5th International Conference on Information, Intelligence, Systems and Applications, pp. 57-62 (2014)

87. Okal, B., Arras, K. O.: Learning Socially Normative Robot Navigation Behaviors with Bayesian Inverse Reinforcement Learning. In: 2016 IEEE International Conference on Robotics and Automation (ICRA), pp. 2889-2895 (2016)

88. Pandey, A., Parhi, D. R.: Multiple Mobile Robots Navigation and Obstacle Avoidance Using Minimum Rule Based ANFIS Network Controller in the Cluttered Environment. International Journal of Advanced Robotics and Automation 1(1), 1-11 (2016)

89. Xia, C., El Kamel, A.: Neural Inverse Reinforcement Learning In Autonomous Navigation. Robotics and Autonomous Systems 84, 1-14 (2016)

90. Villacorta-Atienza, J. A., Makarov, V. A.: Neural Network Architecture for Cognitive Navigation in Dynamic Environments. IEEE Transactions on Neural Networks and Learning Systems 24(12), 2075-2087 (2013) 